\title{
Comprehensive evaluation for the one-pot biosynthesis of butyl-acetate by using microbial mono and co-cultures
}

\author{
Yang $\mathrm{Lv}^{1}$, Yujia Jiang ${ }^{1}$, Jiasheng $\mathrm{Lu}^{1}$, Hao Gao ${ }^{1}$, Weiliang Dong ${ }^{2}$, Jie Zhou ${ }^{1}$, Wenming \\ Zhang $^{2}$, Fengxue Xin ${ }^{2}$, and Min Jiang ${ }^{2}$ \\ ${ }^{1}$ Nanjing Tech University \\ ${ }^{2}$ Nanjing University of Technology
}

May 22, 2021

\begin{abstract}
Butyl acetate has shown wide attention in food, cosmetics, medicine and biofuel sectors. These short chain fatty acid esters can be produced by either chemical or biological synthetic process with corresponding alcohols and acids. Currently, biosynthesis of short chain fatty acid esters, such as butyl butyrate through microbial fermentation systems has been achieved; however, few studies regarding biosynthesis of butyl acetate were reported. In this study, three proof-of-principle strategies for the one-pot butyl acetate production from glucose by microbial fermentation was designed and evaluated. (1) $7.3 \mathrm{~g} / \mathrm{L}$ of butyl acetate was synthesized by butanol producing Clostridium acetobutylicum NJ4 with the supplementation of exogenous acetic acid; (2) With the addition of butanol, $5.76 \mathrm{~g} / \mathrm{L}$ of butyl acetate can be synthesized by acetate producing Actinobacillus succinogenes130z ( $\Delta$ pflA) with the supplementation of exogenous butanol; (3) Microbial co-culture of C. acetobutylicum NJ4 and A. succinogenes130z ( $\Delta$ pflA) can directly produce $2.2 \mathrm{~g} / \mathrm{L}$ of butyl acetate from glucose, representing the first study in terms of butyl acetate production by using microbial co-culture system. Through the immobilization of A. succinogenes $130 \mathrm{z}$ $(\Delta \mathrm{pflA})$, butyl acetate production was further improved to $2.86 \mathrm{~g} / \mathrm{L}$. These strategies may be extended to the biosynthesis of a wide range of esters, especially to some longer chain ones.
\end{abstract}

\section{Hosted file}

Manuscript_12.05.2021.pdf available at https://authorea.com/users/287103/articles/523092comprehensive-evaluation-for-the-one-pot-biosynthesis-of-butyl-acetate-by-using-

microbial-mono-and-co-cultures

\section{Hosted file}

Figures_12.05.2021.pdf available at https://authorea.com/users/287103/articles/523092comprehensive-evaluation-for-the-one-pot-biosynthesis-of-butyl-acetate-by-usingmicrobial-mono-and-co-cultures 